\title{
Diagnosis and surgical management of orbital sparganosis
}

\author{
Qing Xia ${ }^{1} \cdot$ Jianhua Yan ${ }^{1}$
}

Received: 16 April 2018 / Revised: 13 March 2019 / Accepted: 15 March 2019 / Published online: 3 April 2019

(c) The Royal College of Ophthalmologists 2019

\begin{abstract}
Purpose/background Orbital sparganosis represents an extremely rare condition with only a few cases being reported in literature. Here we describe cases of orbital sparganosis, including their etiology, clinical findings, and surgical outcomes. Patients and methods A retrospective review was performed on patients with orbital sparganosis, who were treated at Zhongshan Ophthalmic Center, of Sun Yat-sen University, China between 2000 and 2012.

Results Five patients (three males and two females, one right orbit and four left orbits) were identified. Their mean age was 14.8 years (range $=6-33$ years). Four cases were contracted from eating raw snakes and fishes, or placing poultices of frog on open wounds. All cases showed a swelling and/or redness of the eyelid and conjunctiva, and a migrating inflammation was present in one of the cases. High levels of blood eosinophils were observed in three of these cases. Of the two patients examined using computed tomography, one showed a diffuse soft tissue infiltration and a punctate calcification, while one of the three patients examined using magnetic resonance imaging displayed an annular "tunnel sign" within the lesion. All patients underwent an anterior orbitotomy and the entire worm was removed with no surgical complications.

Conclusions Orbital sparganosis should be highly suspected in patients with a history of eating raw snakes and frogs, a migrating orbital inflammation and the presence of eosinophilia. Orbital imaging examinations play an important role in the diagnosis of orbital sparganosis. Surgical removal of the entire worm is required.
\end{abstract}

\section{Introduction}

Encountering a live worm within the orbit is extremely rare. Human sparganosis is a food-borne zoonosis resulting from the plerocercoid larvae (spargana) of diphyllobothroid tapeworms of the genus Spirometra [1]. While this condition has been documented worldwide, it is mostly reported in east and southeast Asia [1-4]. The spargana can invade the brain, eyes, breasts, pleura, abdominal cavity, bone, genitourinary tract, spinal cord, and subcutaneous tissues, resulting in local tissue damage, paralysis, blindness, and death [1-3]. The clinical features of orbital sparganosis are often not specific and the disease is easily misdiagnosed as involving other orbital lesions, especially idiopathic orbital inflammation [5, 6]. Due to its rare incidence, the diagnosis and surgical treatment of orbital sparganosis are unfamiliar

Jianhua Yan

yanjh2011@126.com

1 The State Key Laboratory of Ophthalmology, Zhongshan Ophthalmic Center, Sun Yat-sen University, 510060 Guangzhou, People's Republic of China to most clinicians. Within the literature, there currently exist only a few cases of orbital sparganosis [3-14]. As a large ophthalmic referral center in southern China, here we report five cases of orbital sparganosis and provide an analysis of its etiology, clinical findings, imaging features, and surgical experience.

\section{Patients and methods}

A retrospective review was performed on five cases of Chinese patients with orbital sparganosis. Patients were treated at the Zhongshan Ophthalmic Center, of Sun Yat-sen University, Guangzhou, China between 1 January 2000 and 31 December 2012. The ethics committee of the Zhongshan Ophthalmic Center approved this retrospective study, which was conducted according to the principles expressed in the Declaration of Helsinki. The committee specifically waived the need for consent. Clinical, operative, and pathological records were reviewed. All patients were seen and treated by a single surgeon (JHY).

A complete medical history and physical examination with laboratory tests, along with an orbital computed tomography (CT) or magnetic resonance imaging (MRI) 
scan were available with a minimum follow-up period of 3 years. Each patient underwent detailed ophthalmologic examinations. In addition to recording basic information on the patient's age, sex, duration of orbital lesion at presentation, ocular data included the affected orbit, laterality, symptoms (visual problem, redness or swelling, proptosis, diplopia and palpable mass), and signs (best-corrected vision, intraocular pressure, anterior segment and fundi of the eye, proptosis, ocular motility deficit, and strabismus) associated with this condition. The etiology of infection, location of the parasite, surgical methods, and complications were recorded in detail. All cases underwent anterior orbitotomy by either skin or conjunctival approach for the removal of the parasite, and the surgical technique was the same as that for other orbital lesions [5-14]. Intravenous dexamethasone $(10 \mathrm{mg} /$ day for adults and $0.25 \mathrm{mg} / \mathrm{kg} /$ day for children) was administered for 3 days post-operatively.

\section{Results}

From an initial retrospective review of 1543 patients with a diagnosis of orbital space-occupied lesions, five patients with orbital sparganosis were identified for inclusion in this report, accounting for $0.32 \%$ of the 1534 orbital lesion patients. The five patients consisted of three males and two females with the right orbit being involved in one patient and the left in four patients. Patients ranged in age from 6 to 33 years with an average age of 14.8 years. Mean follow-up was 4.2 years (range $=3-6$ years).

With the exception of one case where a definitive route of sparganosis infection was not ascertained, all remaining four cases were contracted from ingesting uncooked snake's bile and blood (one case), eating raw fish flesh (one case), frequent catching of frogs (one case), or placing poultices of frog on open wounds (one case). The primary symptoms as observed in all five cases involved a swelling and/or redness of the eyelid and conjunctiva (Fig. 1). Two patients experienced pain in the lesion area, two displayed exophthalmos, and one had limited ocular motility. A migrating inflammation was present in one case. In one case, the subconjunctival worm demonstrated a subconjunctival yellow-white granulomatous lesion along with a conjunctival congestion of the right eye, which represents a relatively characteristic finding for conjunctival sparganosis (Fig. 2). Routine blood analysis showed high levels of blood eosinophils in three of the patients. Following systemic corticosteroid administration, the symptoms and signs partially subsided in all five cases, but after drug
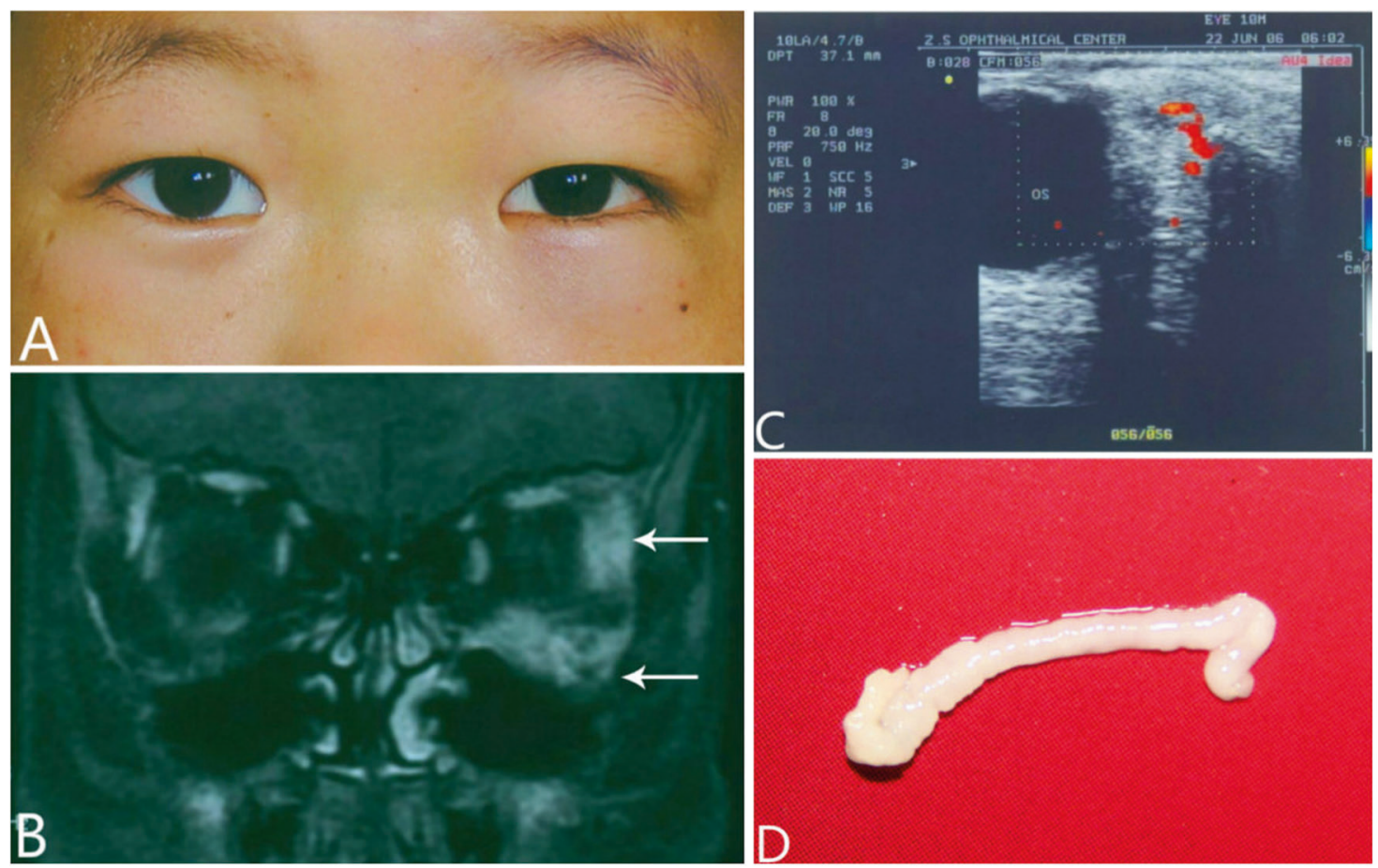

Fig. 1 Case 2, a 9-year-old male with left orbital sparganosis. a Obvious swelling of the left lower eyelid and a non-tender, $25 \mathrm{~mm} \times$ $10 \mathrm{~mm}$ sized, undefined band-shaped mass was located behind the eyelid. b Magnetic resonance imaging (MRI) displayed that the lesion affected both the inferior and lateral regions of the left orbit with an annular "tunnel sign". The lateral and inferior rectus muscles were thickened. c Color doppler ultrasonography revealed a slightly hyperechoic, irregular, and uneven $23 \mathrm{~mm} \times 17 \mathrm{~mm} \times 14 \mathrm{~mm}$ lesion in the lower region of the left orbit, with a moderate blood flow signal inside the mass. d After anterior orbitotomy with skin incision, the entire sparganum $(85 \mathrm{~mm} \times 5 \mathrm{~mm} \times 2 \mathrm{~mm})$ was removed 

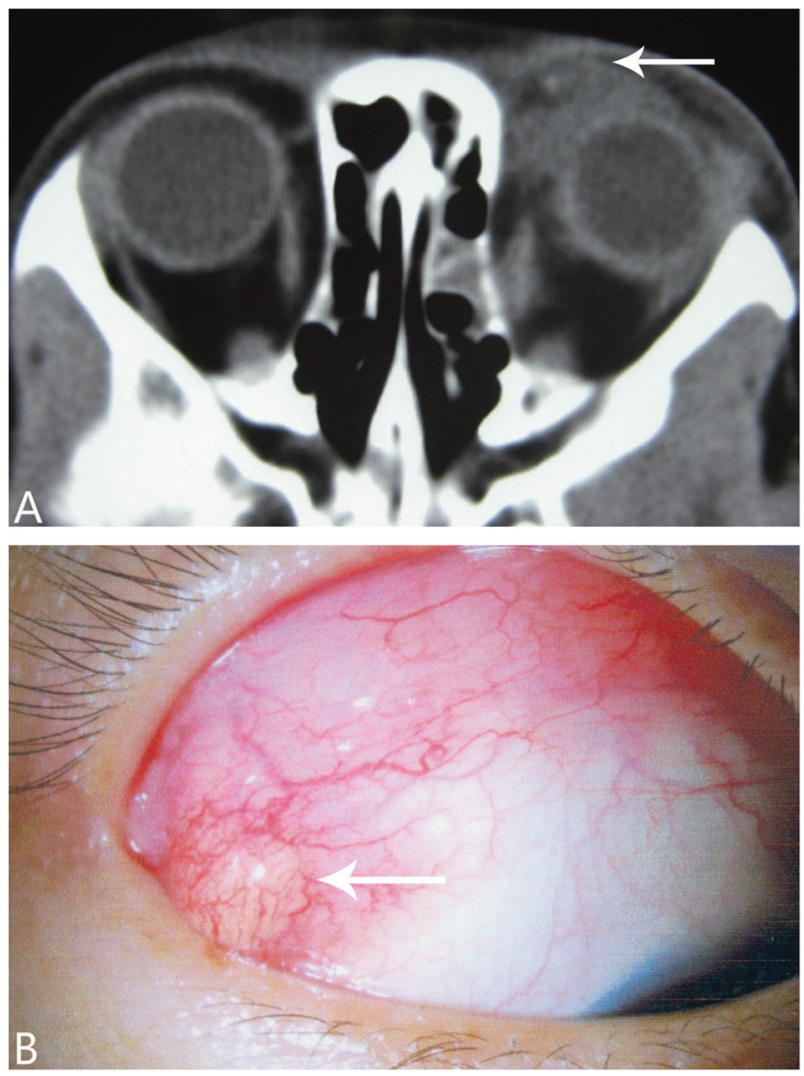

Fig. 2 a Case 1, a 6-year-old female. Computed tomography (CT) scan revealed the obvious swelling of the left upper eyelid, diffuse soft tissue infiltration and a punctate calcification, findings indicating typical features of orbital sparganosis as shown using CT scan. b Case 4, a 33-year-old male patient with a subconjunctival yellow-white granulomatous lesion, along with conjunctival congestion of the right eye indicating a characteristic finding for conjunctival sparganosis

withdrawal all showed a relapse. All patients were misdiagnosed as idiopathic orbital inflammation before surgery.

Imaging techniques including both CT and MRI showed an irregular undefined non-homogeneous infiltration of orbital soft tissue, quite similar to that of an inflammatory lesion. However, within the two cases examined by CT, one showed a diffuse soft tissue infiltration and punctate calcification, which is a relatively typical feature of CT scan for orbital sparganosis (Fig. 2). In three cases examined by MRI, one case displayed an annular "tunnel sign" within the lesion, again a typical feature of MRI for orbital sparganosis (Fig. 1). B-ultrasonography in all cases revealed a hypoechoic solid lesion in the affected area with irregular internal echoes, similar to idiopathic orbital inflammation (Fig. 1).

All cases underwent anterior orbitotomy by a skin (four cases) or conjunctival approach (one case) for complete removal of the worm, with no complications. Only one worm was present in each case and appeared as a white, flat wrinkled string-like parasite (Fig. 1). All removed worms were alive, ranged in size from 7 to $11 \mathrm{~cm}$ and moved slowly. During the surgical procedure, the entire worm was easily removed with the use of forceps. Histopathologic evaluation demonstrated a larva and granulomatous reaction with a substantial amount of eosinophils. Palpebral and conjunctival swellings dissipated after surgery. Detailed investigation revealed no parasitic infection in other parts of the body. A summary of the demographic data, clinical manifestations, imaging features and surgical methods for each case is presented in Table 1.

\section{Discussion}

The lifecycle of Spirometra consists of Spirometra adult worms residing in the intestines of dogs and cats, with their eggs shed in feces and embryonate in the environment. Eggs hatch in water and release coracidia, which are ingested by the first intermediate host cyclops and develop into procercoid larvae. The second intermediate hosts, including frogs and fish, absorb infected copepods enabling procercoid larvae to develop into plerocercoid larvae. When a predator (e.g., dogs or cats) consumes an infected second intermediate host, the lifecycle begins again. Human beings and snakes can serve as the second intermediate host [1]. As spargana reside in frogs and snakes, human beings are mainly infected via consumption of raw or undercooked meat derived from these animals, through drinking untreated water containing Spirometra larvae, or through use of raw flesh as traditional poultices [1, 2]. Ocular sparganosis is quite rare clinically, but accounts for $12.8 \%$ of human sparganosis [2]. In southern China, people exhibit a number of unfortunate habits that can result in orbital sparganosis, including drinking raw snake bile and blood, eating raw fish flesh like Sashimi and treating skin wounds with frog poultices. In our case series, the etiology for four of the five cases was attributable to these habits.

Due to the rare incidence and numerous variations in manifestations, it is difficult to clinically diagnose and differentiate sparganosis from a wide variety of other inflammatory and neoplastic disorders [9]. Depending on the worm's location, the common signs and symptoms in patients with orbital sparganosis include itching, pain, eyelid redness and swelling, exophthalmos, ocular motility limitation, and decreased visual acuity, all of which are nonspecific [3-5]. Eyelid lesions with a mass in the orbit are the most common presentation [5]. Migrating inflammation, a relatively specific finding for orbital worms, occurred in only one case of our series. CT/MRI imaging often reveal orbital inflammatory lesions. The reactive inflammation and recurrent attacks resulting from the parasite are partially relieved after systemic administration of corticosteroids, similar to that observed with idiopathic orbital inflammation. All of our cases showed mild eyelid swelling and inflammation in the anterior orbit, and were thus 


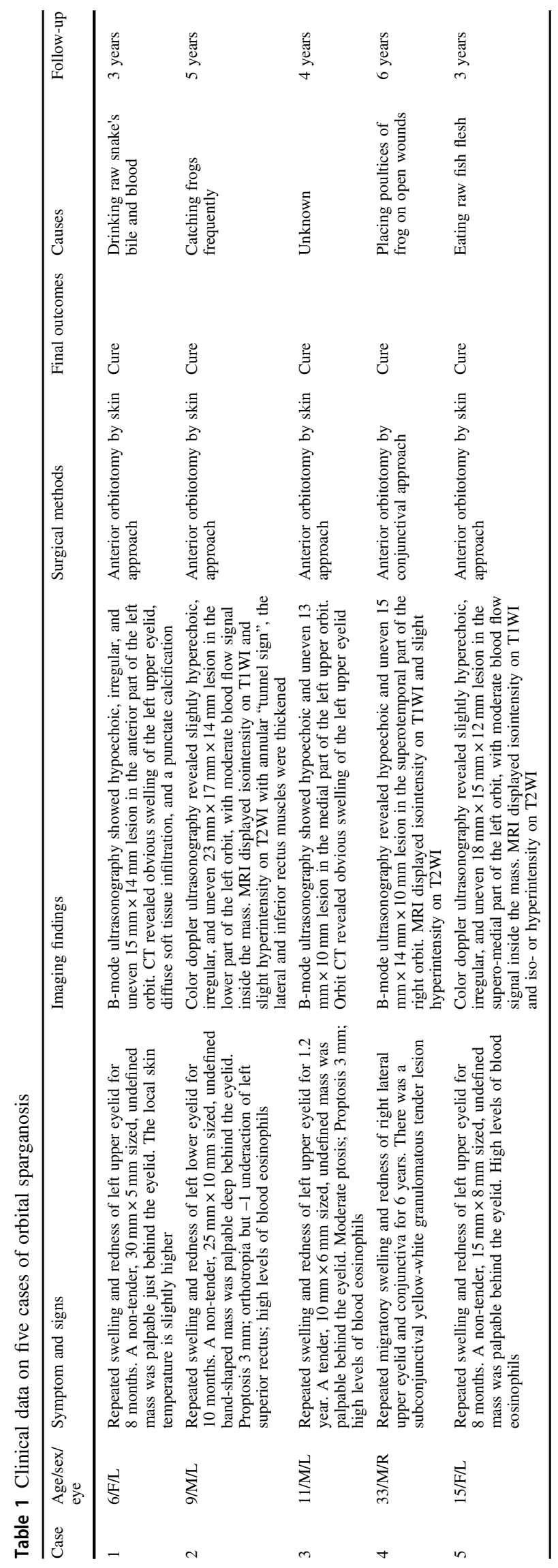

misdiagnosed as idiopathic orbital inflammation. Only after orbital surgical identification of the parasite was a final definitive diagnosis of orbital sparganosis possible. However, with the exception of the presence of a migrating lesion, two important, relatively specific features of parasite infection were ignored in our series. The first was a subconjunctival yellow-white granulomatous lesion present in one case and the second was the observation of high levels of blood eosinophils present in three of the cases.

Occasionally, a preliminary diagnosis of orbital sparganosis can be presumed as based upon findings of abnormal CT, MRI, or ultrasonography [1, 2]. An irregular or nodular-enhanced lesion and a punctate calcification are the typical signs observed with use of CT and were seen in one of our cases. The punctate calcification results from calcium accumulation around the worm. Characteristic lesions as revealed with use of MRI include column or fusiform tunnels with ring-shaped enhancements, which were observed in one of our cases. With regard to ultrasonography, the observation of a subcutaneous eyelid mass presenting as either a linear or column hypoechoic body in the mass may be indicative of orbital sparganosis $[2,5]$. Immunologic serodiagnosis for sparganosis, using antigenspecific IgG antibodies from peripheral blood by enzymelinked immunosorbent assay, has been shown to serve as a beneficial diagnostic tool to complement these imaging findings $[1,9]$. Therefore, based upon the analyses of the cases in this report the following features should alert the clinician to the possibility of this disease: (1) a history of eating raw or undercooked meat derived from snakes or frogs, or using frog meat for skin injury, (2) a migrating inflammatory lesion, (3) a subconjunctival yellow-white granulomatous lesion, along with conjunctival congestion, (4) the detection of eosinophilia, (5) CT scan revealing that eyelid swelling is associated with diffuse soft tissue infiltration and a punctate calcification, and (6) MRI showing an irregular undefined non-homogeneous infiltration of orbital soft tissues with column or fusiform tunnels within the lesion.

Although drugs (praziquantel, albendazole, and mebendazole) can be used for the treatment of human sparganosis, surgical removal of the entire sparganum remains the most effective treatment $[1,2]$. In all our cases, the worm was removed with use of an anterior orbitotomy approach. Only one worm was found in each case and was often wrapped by reactive inflammation in surrounding tissues.

In summary, the clinical manifestations of orbital sparganosis are often not specific and can be similar to that of other chronic inflammations. Due to the rarity of this condition and its capacity to be partially responsive to steroid treatment, it can be easily misdiagnosed as idiopathic orbital inflammation. Orbital lesion patients with a history of eating raw snakes and frogs, or using frog meat for skin injury, 
and/or possessing high levels of eosinophils should be highly suspicious of orbital sparganosis, especially in children showing a migrating inflammation. In such cases, drugs are not effective and only surgical removal can provide a successful resolution. The prognosis of this condition following surgery is quite good.

\section{Summary}

\section{What was known before}

- The clinical manifestations of orbital sparganosis are often not specific and can be similar to that of other chronic inflammations.

\section{What this study adds}

- In this report, we describe cases of orbital sparganosis, including their etiology, clinical findings, imaging features, and surgical outcomes.

Funding This work was supported by the Nature Science Foundation of China (grant number: 81670885), the Science and Technology Program of Guangdong Province, China (gGrant number: 2013B020400003), and by Science and Technology Program of Guangzhou, China (grant number: 15570001). The funders had no role in study design, data collection and analysis, decision to publish, or preparation of the manuscript. No additional external funding was received for this study.

\section{Compliance with ethical standards}

Conflict of interest The authors declare that they have no conflict of interest.
Publisher's note: Springer Nature remains neutral with regard to jurisdictional claims in published maps and institutional affiliations.

\section{References}

1. Quan Liu, Ming-Wei Li, Ze-Dong Wang, Guang-Hui Zhao, XingQuan Zhu. Human sparganosis, a neglected food borne zoonosis. Lancet Infect Dis. 2015;15:1226-35.

2. Li MW, Song HQ, Li C, Lin HY, Xie WT, Lin RQ, et al. Sparganosis in mainland China. Int J Infect Dis. 2011;15:154-6.

3. Zhong HL, Shao L, Lian DR, Deng ZF, Zhao SX, Gao PZ, et al. Ocular sparganosis caused blindness. Chin Med J. 1983;96:73-5.

4. Mentz MB, Procianoy F, Maestri MK, Rott MB. Human ocular sparganosis in southern Brazil. Rev Inst Med Trop Sao Paulo. 2011;53:51-3.

5. Ho TH, Lin MC, Yu WW, Lai PH, Sheu SJ, Bee YS. Ocular sparganosis mimicking an orbital idiopathic inflammatory syndrome. Orbit. 2013;32:395-8.

6. Preechawat P, Poonyathalang A, Panyakorn S, Dekumyoy P. Sparganosis mimicking an orbital myositis. J Neuroophthalmol. 2011;35:219-21.

7. Ausayakhun S, Siriprasert V, Morakote N, Taweesap K. Ocular sparganosis in Thailand. Southeast Asian J Trop Med Public Health. 1993;24:603-6.

8. Subudhi BN, Dash S, Chakrabarty D, Mishra DP, Senapati U. Ocular sparganosis. J Indian Med Assoc. 2006;104:529-30.

9. Yoon KC, Seo MS, Park SW, Park YG. Eyelid sparganosis. Am J Ophthalmol. 2004;138:873-5.

10. Xie X, Hu J, Sun G, Ding B, Feng L. Orbital sparganosis in an 8-year boy: a case report. BMC Ophthalmol. 2018;18:13.

11. Yang JW, Lee JH, Kang S. A case of ocular sparganosis in Korea. Korean J Ophthalmol. 2007;21:48-50.

12. Kubota T, Itoh M. Sparganosis associated with orbital myositis. Jpn J Ophthalmol. 2007;51:311-2.

13. Botterel F, Bourée P. Ocular sparganosis: a case report. J Travel Med. 2003;10:245-6.

14. Kittiponghansa S, Tesana S, Ritch R. Ocular sparganosis:a cause of subconjunctival tumor and deafness. Trop Med Parasitol. 1988;39:247-8. 